\title{
Benign vs malignant inferolateral early repolarization: Focus on the T wave
}

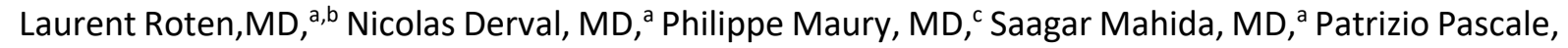
MD, ${ }^{a}$ Antoine Leenhardt, MD, ${ }^{d}$ Laurence Jesel, MD, ${ }^{e}$ Isabel Deisenhofer, MD, ${ }^{f}$ Josef Kautzner, MD, PhD, ${ }^{\mathrm{g}}$ Vincent Probst, $M D{ }^{h}$ Anne Rollin, $M D,{ }^{c}$ Jean-Bernard Ruidavets, $M D,{ }^{c}$ Jean Ferrières, MD, ${ }^{i}$ Frédéric Sacher, MD, ${ }^{a}$ Dik Heg, MD, PhD, ${ }^{j}$ Daniel Scherr, MD, FHRS, ${ }^{a}$ Yuki Komatsu, MD, ${ }^{a}$ Matthew Daly, MD, ${ }^{a}$ Arnaud Denis, $M D,{ }^{a}$ Ashok Shah, MD, ${ }^{\text {a }}$ Mélèze Hocini, MD, ${ }^{\text {a }}$ Pierre Jaïs, MD, ${ }^{\text {a }}$ Michel Haïssaguerre, $M D^{\mathrm{a}}$

${ }^{a} \mathrm{CHU}$ de Bordeaux/IHU Institut de Rythmologie et Modélisation Cardiaque, Université Bordeaux/Inserm U1045, Bordeaux, France

${ }^{\mathrm{b} D e p a r t m e n t}$ of Cardiology, Inselspital, Bern University Hospital, and University of Bern, Bern, Switzerland

${ }^{c}$ Centre Hospitalier Universitaire de Toulouse, Toulouse, France

dDépartement de Cardiologie et Centre de Référence des Maladies Cardiaques Héréditaires, AP-HP, Hôpital Bichat, Université Paris Diderot, Sorbonne Paris Cité, Paris, France

${ }^{\text {e}}$ Centre Hospitalier Universitaire de Strasbourg, Strasbourg, France,

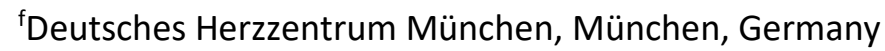

IInstitute for Clinical and Experimental Medicine, Prague, Czech Republic

h''institut du thorax, service de cardiologie du CHU de Nantes, Nantes, France

'Department of Cardiology, Toulouse University School of Medicine, Toulouse, France

${ }^{\mathrm{j} C T U}$ Bern, Department of Clinical Research, and Institute of Social and Preventive Medicine (ISPM), University of Bern, Bern, Switzerland

${ }^{*}$ Correspondence: Dr. LaurentRoten, Kardiologie, Inselspital, Freiburgstrasse, 3010 Bern, Switzerland. E-Mail address: laurent.roten@insel.ch 
This work was supported by special grants from the Direction Générale de l'Offre de Soins (PHRC No. 20-12) and through the Investment of the Future Grant ANR-10-IAHU-04 from the government of France through the Agence National de la Recherche.

\section{Tables and 5 Figures}

Table 1: Baseline ECG characteristics

Table 2: Performance of various ECG parameters in differentiating malignant and benign inferolateral ER

Table 3: Binomial logistic regression model

Figure 1: Two examples showing how to calculate the $T / R$ ratio

Figure 2: A: Bar graph showing the distribution of QTc interval (in milliseconds) for the ventricular fibrillation (VF)group (green bars) and controls (blue bars). B: Dot plot of the lower T/R ratio (lead II or $V_{5}$ ) for the VF group and controls.

Figure 3: ST-segment morphology measured $100 \mathrm{~ms}$ after the J point (ST $\leq 0.0 \mathrm{mV}, \mathrm{ST} \leq 0.05 \mathrm{mV}, \mathrm{ST} \leq$ $0.1 \mathrm{mV}$, and ST $>0.1 \mathrm{mV}$ ) and corresponding T-wave amplitudes in leads II and $\mathrm{V}_{5}$ for the ventricular fibrillation (VF) group and controls.

Figure 4: Receiver operating characteristic curves for differentiating malignant from benign inferolateral early repolarization based on maximal J-wave amplitude, QTc interval, and lower $T / R$ ratio (lead II or $\left.V_{5}\right) . A U C=$ area under receiver operating characteristic curve

Figure 5: ECG examples of inferolateral early repolarization 
ABSTRACT

Background: Inferolateral early repolarization (ER) is highly prevalent and is associated with idiopathic ventricular fibrillation(VF).

Objective: The purpose of this study was to evaluate the potential role of T-wave parameters to differentiate between malignant and benign ER.

Methods: We compared the ECGs of patients with ER and VF $(n=92)$ with control subjects with asymptomatic ER $(n=247)$. We assessed J-wave amplitude, QTc interval, T-wave/R-wave $(T / R)$ ratio in leads II and V5, and presence of low-amplitude T waves (T-wave amplitude $<0.1 \mathrm{mV}$ and $<10 \%$ of R-wave amplitude in lead I, II, or V4-V6).

Results: Compared to controls, the VF group had longer QTc intervals (388 ms vs $377 \mathrm{~ms}, \mathrm{P}=.001$ ), higher J-wave amplitudes ( $0.23 \mathrm{mV}$ vs $0.17 \mathrm{mV}, \mathrm{P}<.001)$, higher prevalence of low-amplitude T waves ( $29 \%$ vs $3 \%, \mathrm{P}<.001)$, and lower $\mathrm{T} / \mathrm{R}$ ratio $(0.18$ vs $0.30, \mathrm{P}<.001)$. Logistic regression analysis demonstrated that QTc interval (odds ratio [OR] per 10 ms: 1.15, 95\% confidence interval [Cl\} 1.021.30), maximal J-wave amplitude (OR per $0.1 \mathrm{mV}: 1.68,95 \% \mathrm{Cl}$ 1.23-2.31), lower $\mathrm{T} / \mathrm{R}$ ratio (OR per 0.1 unit: $0.62,95 \% \mathrm{Cl} 0.47-0.81$ ), presence of low-amplitude T waves (OR 3.53, 95\% Cl 1.26-9.88), and presence of J waves in the inferior leads (OR 2.58, 95\% $\mathrm{Cl} 1.18-5.65)$ were associated with malignant ER.

Conclusions: Patients with malignant ER have a higher prevalence of low-amplitude T waves, lower T/R ratio (lead II or V5), and longer QTC interval. The combination of these parameters with J-wave amplitude and distribution of J waves may allow for improved identification of malignant ER. Keywords: J wave; Early repolarization; Ventricular fibrillation; Electrocardiogram; QT interval Abbreviations: $\mathrm{Cl}$ = confidence interval; $\mathrm{ECG}$ = electrocardiogram; $\mathrm{ER}=$ early repolarization; $\mathrm{OR}$ = odds ratio; $\mathrm{VF}$ = ventricular fibrillation 


\section{INTRODUCTION}

The electrocardiographic (ECG) pattern of inferolateral early repolarization (ER) is common, with a particularly high prevalence reported among athletes and adolescents. ${ }^{1}$ An association between inferolateral ER with sudden cardiac arrest has been established by a number of different groups. ${ }^{2}$ Population-based studies have also reported an increased mortality among patients with inferolateral ER compared to controls. ${ }^{1,3,4}$ Despite the reports linking ER with sudden death, only a small minority of patients with this pattern on the ECG will have sudden cardiac arrest, while the majority remain asymptomatic. The identification of this minority of patients represents a significant challenge. Currently, the identification of the malignant variant of the ER pattern is reliant on parameters such as the J-wave distribution, J-wave amplitude, and ST-segment morphology. ${ }^{5,6}$ However, the sensitivity and specificity of these parameters remain limited. Additionally, assessment of ST-segment morphology is difficult. The T wave may provide similar information as the ST-segment morphology measured at 100 ms after the J point, and other shave advocated analysis of repolarization markers independently of J-wave amplitude for risk stratification in inferolateral ER. ${ }^{7}$ Furthermore, the concomitant presence of inferolateral ER and long QT syndrome seems to increase arrhythmic risk. ${ }^{8,9}$ In this study, we sought to determine the potential role of T-wave parameters to differentiate malignant and benign forms of inferolateral ER. 


\section{METHODS}

\section{Study population}

Cases with ER and aborted sudden death were included from the International Registry of Idiopathic Ventricular Fibrillation, which has enrolled ventricular fibrillation (VF) patients from various tertiary care arrhythmia centers since January $2007 .^{2}$ The diagnosis of idiopathic VF for patients included in the registry is based on the absence of identifiable structural heart disease (normal echocardiography) and detectable coronary artery disease (normal exercise testing or normal coronary angiography). Exclusion criteria for the registry include a corrected QT interval (QTc) $<340 \mathrm{~ms}$ or $>440 \mathrm{~ms}$, spontaneous or drug-induced Brugada type ${ }^{1}$ ECG pattern, and catecholaminergic polymorphic ventricular arrhythmia.

For the purposes of this study, we included patients with idiopathic VF with inferolateral ER (VF group) and an ECG of suitable quality for detailed analysis. The diagnosis of inferolateral ER was based on elevation of the QRS-ST junction (J point) by $\geq 0.1 \mathrm{mV}$ above baseline in $\geq 2$ contiguous inferior (II, III, $\mathrm{aVF})$ and/or lateral leads $\left(\mathrm{I}, \mathrm{aVL}\right.$, and $\left.\mathrm{V}_{4}-\mathrm{V}_{6}\right)$. The J-point elevation manifested as either $\mathrm{QRS}$ slurring or notching. A total of 92 patients from the registry fulfilled these criteria. Importantly, 8 patients with ER and idiopathic VF could not be included in the registry because of QTc $>440 \mathrm{~ms}$ ( $\mathrm{n}=7$; median QTc interval $470 \mathrm{~ms}$, range $456-476 \mathrm{~ms}$ ) or $<340 \mathrm{~ms}$ ( $\mathrm{n}=1$; QTc $310 \mathrm{~ms}$ ).

The control group consisted of subjects from the third Toulouse MONICA survey $(n=1171)^{10,11}$ and a subsample of the MONALISA study (study for Monitoring NAtionaL du rISque Artériel; $\mathrm{n}=751$ )..$^{12,13}$ The subjects of these studies were middle-aged men and women living in south-western France. They were randomly recruited from the general population. The objectives were to measure trends in cardiovascular mortality, coronary heart disease, and cerebrovascular disease morbidity. ${ }^{10,12,13}$ All subjects of these studies were included as controls irrespective of outcome during follow-up if they had inferolateral ER and an ECG of suitable quality for analysis $(n=250)$. One control subject with inferolateral ER was excluded because of QTc $>440 \mathrm{~ms}$ (QTc $453 \mathrm{~ms}$ ), and 2 control subjects were 
excluded due to QTc $<340 \mathrm{~ms}$ (QTc $336 \mathrm{~ms}$ and $332 \mathrm{~ms}$, respectively). The final control group consisted of a total of 247 subjects.

The study complies with the Declaration of Helsinki and was approved by the respective institutional review boards at all participating centers.

\section{ECG analysis}

All ECGs were digitized and analysed with a digital caliper (Iconico, Cardio Calipers, www.iconico.com).

ECGs without a scale and those of low quality precluding any analysis were excluded. If several ECGs remained, ECGs recorded close to the arrhythmic event (usually within 1 week) were discarded. Of the remaining ECGs, the ECG with the highest J-wave amplitude was chosen for analysis. Median time from VF to the ECG chosen for analysis was 20 days. Heartrate, PR interval, QRS width, and QT interval were measured. The Bazett formula was used to correct QT interval for heart rate (QTc). Inferior (II, III, aVF), high lateral (I, aVL), and lateral $\left(\mathrm{V}_{4}-\mathrm{V}_{6}\right)$ leads were analysed for the presence of $\mathrm{J}$ waves. Overall J-wave morphology was assessed as either only slurred J waves or any notched J wave (presence of only notched, or both notched and slurred J waves). In case of QRS slurring, the J-wave amplitude was measured at the point where slurring started to separate from the descending limb of the R wave and in case of QRS notching, at the top of the notch relative to the baseline. The baseline was defined as the isoelectric line between 2 T-P intervals.

We analysed the ST segment $100 \mathrm{~ms}$ after the J point in leads II and $V_{5}$ if a J wave was present in the respective lead. If the ST-segment amplitude was $>0.1 \mathrm{mV}$, the ST segment was described as ascending/upsloping(ST >0.1 mV); if it was $\leq 0.1 \mathrm{mV}$, it was described as horizontal/descending (ST $\leq 0.1$ $\mathrm{mV}$ ). If the ST segment showed a high take off at the J point and remained elevated $>0.1 \mathrm{mV} 100 \mathrm{~ms}$ after the J point, it also met the definition of ascending/upsloping. Additionally, we analysed whether the ST-segment amplitude was $\leq 0.05 \mathrm{mV}$ (ST $\leq 0.05 \mathrm{mV}$ ) or $\leq 0.0 \mathrm{mV}$ (ST $\leq 0.0 \mathrm{mV})$. We assessed all ECGs for the presence of low-amplitude $T$ waves (dysmorphic $T$ waves). A lowamplitude $T$ wave was defined as any $T$ wave in lead $\mathrm{I}$, II, or $\mathrm{V}_{4}-\mathrm{V}_{6}$ that was either inverted, biphasic, 
or had an amplitude that was both $\leq 0.1 \mathrm{mV}$ and $\leq 10 \%$ of the R-wave amplitude in the same lead.

Amplitudes of $R$ and $T$ waves were measured in leads $\|$ and $V_{5}$, and the $T / R$ ratio was calculated separately for each lead (Figure 1).

\section{Statistical analysis}

Categorical variables are expressed as number and percentage and continuous variables as mean \pm

SD. Categorical variables were compared using the $\chi 2$ test or Fisher exact test and continuous variables

with the unpaired $t$ test. The Kolmogorov-Smirnov test with Lilliefors correction was used for normality testing of the QTc interval within each of the 2 groups. The sensitivity, specificity, positive and negative likelihood ratios, diagnostic odds ratios, and C statistics of various ECG parameters in differentiating malignant and benign inferolateral ER were calculated. Correlation analysis of STsegment morphology with T-wave amplitude was performed with the Spearman correlation coefficient $\left(r_{s}\right)$ and correlation analysis of R-wave amplitude with T-wave amplitude with the Pearson correlation coefficient $\left(r_{p}\right)$. The effect of age on QTc interval and lower T/R ratio (lead II or $V_{5}$ ) was assessed with linear regression analysis. To assess the effect of age on maximal J-wave amplitude, the latter was dichotomized ( $\leq 0.2 \mathrm{mV}$ vs $40.2 \mathrm{mV})$, and a binomial logistic regression analysis performed. A 2-way analysis of variance was performed to determine whether there was an interaction between groups and gender on QTc interval and lower T/R ratio (lead II or $\mathrm{V}_{5}$ ). To assess the interaction between groups and gender on maximal J-wave amplitude, a binomial logistic regression analysis was performed on the dichotomized variable $(\leq 0.2 \mathrm{mV} v s>0.2 \mathrm{mV})$. A binomial logistic regression was performed to ascertain the effect of QTc interval, maximal J-wave amplitude, lower T/R ratio (lead II or $\mathrm{V}_{5}$ ), presence of a dysmorphic T wave, and presence of J waves in the inferior leads on the likelihood of subjects being in the VF group. A 2-sided $P<.05$ was considered significant. All analyses were performed using SPSS 21.0 (SPSS Inc, Chicago, IL). 
Patients in the VF group were significantly younger than controls $(37.1 \pm 13.1$ years vs $50.4 \pm 10.9$ years, $P<.001)$. In both groups, the majority were men (75\% VF group vs $77 \%$ controls; $P \pm .71)$. Heart rate was significantly higher and QTc interval longer in the VF group compared to controls (Table 1).

Of note, although the QTC interval was normally distributed in controls $(P \pm .20$ according to the Kolmogorov-Smirnov test), this was not the case in the VF group ( $P \pm .007$; Figure 2$)$.

\section{J wave}

ER was significantly more prevalent in the inferior leads in the VF group compared to controls (Table 1). However, the prevalence was not different among the 2 groups in the high lateral and lateral leads. The maximal J-wave amplitude was significantly higher in the VF group (Table 1). Specifically, maximal J-wave amplitudes were higher in the inferior and lateral leads in the VF group but were not different in the high lateral leads.

\section{T wave}

Low-amplitude T waves were observed significantly more frequently in the VF group and very rarely in controls (Table 1 ). The T/R ratio in leads II and $V_{5}$ was significantly lower in the VF group (Table 1$)$. This was driven by a lower T-wave amplitude in lead $\mathrm{V}_{5}$ and by a combination of a lower T-wave amplitude and a higher R-wave amplitude in lead II. Figure 2 illustrates the dot plot of the lower T/R ratio (lead II or $\mathrm{V}_{5}$ ) for the 2 groups.

The ST segment following the J wave in lead II was not different among groups. However, in lead $V_{5}$ in the VF group the ST segment following the J wave was significantly less ascending (Table 1). Figure 3 illustrates the correlation of different ST-segment morphologies (ST $\leq 0.0 \mathrm{mV}, \mathrm{ST} \leq 0.05 \mathrm{mV}, \mathrm{ST} \leq 0.1$ $\mathrm{mV}, \mathrm{ST}>0.1 \mathrm{mV}$ ) with T-wave amplitude in lead II (VF group: $r_{\mathrm{s}}=0.54, P<.001$; control group: $r_{\mathrm{s}}=0.48$, 
$P<.001)$ and lead $V_{5}$ (VF group: $r_{s}=0.51, P<.001$; control group: $\left.r_{s}=0.60, P<.001\right)$. R-wave amplitude correlated with T-wave amplitude in lead II (VF group: $r_{p}=0.35, p=0.001$; control group: $r_{p}=0.43$, $P<.001)$ and lead $V_{5}$ (VF group: $r_{p}=0.42, P<.001$; control group: $\left.r_{p}=0.57, P<.001\right)$.

\section{Effect of age and gender}

Regression analysis did not demonstrate a significant effect of age on QTc interval, maximal J-wave amplitude ( $\leq 0.2 \mathrm{mV} v \mathrm{~s}>0.2 \mathrm{mV}$ ), and lower T/R ratio (lead II or V5) for the VF group and controls (see Online Supplementary Table 1).

The analysis also showed no statistically significant interaction between gender and group on QTC interval, maximal J-wave amplitude ( $\leq 0.2 \mathrm{mV}$ vs $>0.2 \mathrm{mV}$ ), and lower T/R ratio (lead II or $\mathrm{V}_{5}$ ) (see Online Supplementary Table 2). There was no statistically significant difference between males and females for maximal J-wave amplitude ( $\leq 0.2 \mathrm{mV}$ vs $>0.2 \mathrm{mV}$ ) and lower T/R ratio (lead II or $\mathrm{V}_{5}$ ). There was a statistically significant difference between males and females for QTc interval and between VF group and controls for QTc interval, maximal J-wave amplitude ( $\leq 0.2 \mathrm{mV} v \mathrm{~s}>0.2 \mathrm{mV}$ ), and lower T/R ratio (lead II or $\mathrm{V}_{5}$ ).

\section{Best performing ECG parameters}

The lower $\mathrm{T} / \mathrm{R}$ ratio (in either lead II or $\mathrm{V}_{5}$ ) was superior to lower T-wave amplitude (in either lead II or $\mathrm{V}_{5}$ ), maximal $\backslash$-wave amplitude, or QTc interval in differentiating malignant from benign ER ( $\mathrm{C}$ statistic $0.77,0.68,0.65$, and 0.61 , respectively; Figure 4). Table 2 demonstrates the performance of the various ECG parameters in terms of identification of malignant inferolateral ER.

The logistic regression model was statistically significant $[\chi 2(5)=85.218, P<.001$; Table 3$]$. The model explained $32 \%$ (Nagelkerke R2) of the variance among groups and correctly classified $79 \%$ of all subjects. Sensitivity was $38 \%$, and specificity was $94 \%$.

Some representative ECG examples of inferolateral ER in the VF group and in controls are shown in Figure 5. 


\section{DISCUSSION}

Patients with malignant inferolateral ER have longer QTc intervals, a higher prevalence of lowamplitude $T$ waves, and lower $T / R$ ratios in leads II and $V_{5}$ than controls with benign inferolateral ER. These T-wave parameters have superior performance in differentiating malignant from benign inferolateral ER than conventional ECG risk markers such as J-wave distribution, maximal J-wave amplitude, and ST-segment morphology.

Multiple studies have reported that the presence of inferior or a combination of inferior and lateral J waves portends a higher arrhythmic risk compared to lateral J waves inisolation. ${ }^{1,3,4}$ Similarly, higher J-wave amplitudes have been associated with an increased risk of malignant arrhythmias. ${ }^{1-4}$ In keeping with these observations, we observed a higher prevalence of inferior J waves in the VF group compared to controls. We also observed higher maximal J-wave amplitudes in the VF group. Of note, however, control subjects also had a high prevalence of inferior J waves, and there was considerable overlap of maximal J-wave amplitudes between cases and controls.

A horizontal/descending ST-segment morphology has also been reported to be a marker of increased arrhythmic risk in patients with inferolateral ER. ${ }^{5,6,11}$ Consistent with previous reports, we noted a less ascending ST-segment morphology in ER patients with VF compared to control subjects. However, the benign variant, which is characterized by an ascending ST-segment morphology ( $40.1 \mathrm{mV}, 100 \mathrm{~ms}$ after the J point), was rare even among controls; therefore, its specificity is poor. We also analysed an intermediate form of ascending ST-segment morphology (40.05 mV, $100 \mathrm{~ms}$ after the J point). However, this did not improve the discriminatory performance of the ST-segment morphology.

It is important to note that characterizing the ST-segment morphology has drawbacks. For instance, the definition is not uniform. There is no consensus as to whether only leads with a J wave should be assessed, whether the predominant pattern should be reported, or whether the observation of a horizontal ST-segment morphology in a single lead is sufficient to classify the pattern as malignant. Furthermore, it is difficult to assess whether ST-segment amplitude is $40.1 \mathrm{mV} 100 \mathrm{~ms}$ after the J point. 
In contrast to the drawbacks related to defining ST-segment morphology, measuring the T/R ratio is straight forward. A tall T wave usually is preceded by a more ascending ST-segment morphology. Accordingly, we observed a good correlation between ST-segment morphology and T-wave amplitude. As shown in our study, T-wave amplitude is also correlated to R-wave amplitude. Therefore, it seems reasonable to determine the relationship of the T-wave amplitude to the preceding R-wave amplitude. Compared to $T$-wave amplitude, the $T / R$ ratio also demonstrated superior performance in differentiating malignant from benign inferolateral ER. Hence, ease of measurement and the possibility to correct the T-wave amplitude based on the R-wave amplitude are important advantages compared to ST-segment morphology. Therefore, we advocate the replacement of ST-segment morphology by lower T/R ratio (lead II or $\mathrm{V}_{5}$ ) for risk stratification in inferolateral ER. Another important finding of our study is a longer QTc interval among patients in the VF group, with an associated non gaussian distribution. More specifically, we observed several individuals with a QTC interval at the upper limit of normal in the VF group, whereas only a few control subjects had QTc intervals at the upper normal limit, eventhough controls were 2.5 times more numerous. This finding is reinforced by the fact that QTc interval 4440 ms was an exclusion criterion for our registry. Accordingly, 7 patients were not included in the registry because of QTc interval $4440 \mathrm{~ms}$. In contrast, only 1 subject in the control group had to be excluded because of QTc interval 4440 ms, although the control group consisted of all patients within ferolateral ER from the French population-based MONICA survey and a subsample of the MONALISA study in southwestern France. Previous studies have reported that the QTc interval has anormal distribution in the general population. Consistent with these reports, the QTc intervals of our control cohort showed the expected Gaussian distribution (Figure 2). Compared to subjects without inferolateral ER, both healthy subjects with inferolateral ER ${ }^{14}$ and patients with malignant inferolateral $E \mathrm{R}^{15}$ have been reported to have shorter QTc intervals. In this study however, we compared QTc intervals among groups both having inferolateral ER and found longer QTc intervals in the VF group. Nevertheless, mean QTc intervals were rather short in both 
groups (388 ms and 377 ms for VF group and controls, respectively) compared to published values in healthy controls without inferolateral ER; therefore, this finding is not contradictory. ${ }^{15}$

In addition to studies linking ER in healthy subjects with an increased risk of malignant arrhythmia, multiple studies have reported that ER is a modulator of arrhythmic risk in patients with cardiac disease. ${ }^{16}$ In patients with long QT syndrome, the presence of inferolateral ER has been demonstrated to increase the probability of adverse events. ${ }^{9}$ In a Canadian registry of patients with apparently unexplained sudden cardiac arrest, further workup yielded a diagnosis in $44 \%$ of patients. ${ }^{8}$ Long QT syndrome was the most common diagnosis. Interestingly, the prevalence of inferolateral ER in this study was $23 \%$, both in patients with long QT syndrome and in patients with idiopathic VF. Our findings provide further corroborating evidence implicating inferolateral ER as a modulator of risk in patients with subtle variations in QTC interval. Overall, our findings and those of others suggest that the concurrent presence of inferolateral ER and a QTc interval at the upper normal limit might be an ominous combination.

According to our model, T-wave parameters such as the presence of dysmorphic T waves and a low $T / R$ ratio (lead II or $V_{5}$ ) are associated with malignant inferolateral ER. Additionally, QTc intervals at the upper limit of normal are rarely seen in controls with inferolateral ER. Therefore, we propose combining these T-wave parameters with the traditional parameters, that is, maximal J-wave amplitude and J-wave distribution, to enhance risk stratification in patients with inferolatera I ER. Depending on the clinical situation, one may choose different cut off values of those variables to have either a high sensitivity or a high specificity.

\section{STUDY LIMITATIONS}

Malignant inferolateral ER is a rare disease. Cases in this study are over represented, which may have affected the model. However, calculations of sensitivity and specificity typically are not affected by an incorrect representation of prevalence in case-control studies. The cases of our registry on idiopathic VF originate from multiple centers around the world. We have limited control on patients election in 
both idiopathic VF patients and in the control population, which might have introduced some selection bias. Some patients in the VF group might have unrecognized, limited structural heart disease, which can be responsible for VF. On the other hand, structural heart disease and idiopathic VF maybe present in some control subjects. The QTc interval may have been overestimated in the VF group because of a higher heart rate in this group and under correction by the Bazett formula. Finally, the patients in the VF group were significantly younger than controls, although we did not detect an effect of age on the main variables investigated in this study.

301

\section{CONCLUSION}

Patients with malignant ER have a higher prevalence of low-amplitude T waves, lower T/R ratio (lead II or $V_{5}$ ), and longer QTc interval, which lacks atypical gaussian distribution. Combining these parameters with maximal J-wave amplitude and presence of $\mathrm{J}$ waves in the inferior leads may allow for improved identification of malignant ER.

\section{ACKNOWLEDGMENTS}

The following physicians kindly contributed to data collection: Gabriel Laurent (Dijon), Pascal Defaye (Grenoble), Dominique Lacroix (Lille), Maurice Pornin (Paris), Frederic Anselme (Rouen), Patrice Scanu (Caen), Paul Bru (La Rochelle), Nicolas Delarche (Pau), Jean Vidal (Niort), Pascal Chavernac (Castres), (Montpellier), Christian de Chillou (Nancy), Elisabeth Somody (Montauban), Philippe Jarnier (Perigueux), Michel Lopez (Lyon), Aude Tassin (Angers), Pierre Winum (Nimes), Georges Nadji (Perpignan), Sinikka Yli-Mayry (Tampere), Nohiriro Komiya (Nagasaki), Yoshifusa Aizawa (Niigata), Akihiko Nogami (Tsukuba), Thomas Arentz (Bad Krozingen), Martin Borggrefe (Mannheim), Thomas Rostock (Mainz), Roberto Mantovan (Treviso), Riccardo Cappato (Milan), Luc de Roy, (Leuven), Piotr Platonov (Lund), Anders Englund (Örebro), and Jürg Schläpfer (Lausanne). 


\section{CLINICAL PERSPECTIVES}

Inferolateral early repolarization has a high prevalence but is also associated with ventricular fibrillation. Risk stratification remains challenging, especially in subjects with syncope or positive family history for sudden cardiac death. Traditional markers of malignant inferolateral early repolarization are J-wave amplitude, J-wave distribution, and horizontal ST- segment morphology, but performance of these markers is modest. This study puts the focus of risk stratification for malignant early repolarization on the $T$ wave. It introduces the concept of dysmorphic $T$ waves and $T / R$ ratio in leads II and $V_{5}$. Subjects with malignant inferolateral early repolarization have a higher prevalence of dysmorphic $T$ waves, lower T/R ratio in leads II and $V_{5}$, and longer QTc intervals compared to healthy controls with inferolateral early repolarization. These new markers, together with traditional marker so far rhythmic risk, may help improve risk stratification of inferolateral early repolarization. Before clinical application, the findings of this case-control study need further verification in large-scale population studies.

\section{SUPPLEMENTARY INFORMATION}

Supplementary data associated with this article can be found in the online version at http://dx.doi.org/10.1016/j.hrthm. 2015.11.020.

\section{REFERENCES}

1. Tikkanen JT, Anttonen O, Junttila MJ, Aro AL, Kerola T, Rissanen HA, Reunanen A, Huikuri HV. Long-term outcome associated with early repolarization on electrocardiography. $\mathrm{N}$ Engl J Med 2009;361:2529-2537.

2. Haissaguerre $M$, Derval $N$, Sacher $F$, et al. Sudden cardiac arrest associated with early repolarization. N Engl J Med 2008;358:2016-2023.

3. Haruta D, Matsuo K, Tsuneto A, Ichimaru S, Hida A, Sera N, Imaizumi M, Nakashima E, Maemura $\mathrm{K}$, Akahoshi $\mathrm{M}$. Incidence and prognostic value of early repolarization pattern in the 12-lead electrocardiogram. Circulation 2011;123: 2931-2937.

4. Sinner MF, Reinhard W, Muller M, et al. Association of early repolarization pattern on ecg with risk of cardiac and all-cause mortality: a population-based prospective cohort study (MONICA/KORA). PLoS Med 2010;7:e1000314. 
5. Tikkanen JT, Junttila MJ, Anttonen O, Aro AL, Luttinen S, Kerola T, Sager SJ, Rissanen HA, Myerburg RJ, Reunanen A, Huikuri HV. Early repolarization: electrocardiographic phenotypes associated with favourable long-term outcome. Circulation 2011;123:2666-2673.

6. Rosso R, Glikson E, Belhassen B, Katz A, Halkin A, Steinvil A, Viskin S. Distinguishing "benign" from "malignant early repolarization": the value of the ST-segment morphology.Heart Rhythm 2012;9:225-229.

7. Viskin S, Rosso R, Halkin A. Making sense of early repolarization. HeartRhythm 2012;9:566-568.

8. Derval N, Simpson CS, Birnie $\mathrm{DH}$, et al. Prevalence and characteristics of early repolarization in the Casper registry: cardiac arrest survivors with preserved ejection fraction registry. J Am Coll Cardiol 2011;58:722-728.

9. Laksman ZW, Gula LJ, Saklani P, Cassagneau R, Steinberg C, Conacher S, Yee R, Skanes A, LeongSit $P$, Manlucu J, Klein GJ, Krahn AD. Early repolarization is associated with symptoms in patients with type 1and type 2 long qt syndrome. Heart Rhythm 2014;11:1632-1638.

10. Kuulasmaa K, Tunstall-Pedoe H, Dobson A, Fortmann S, Sans S, Tolonen H, Evans A, Ferrario M, Tuomilehto J. Estimation of contribution of changes in classic risk factors to trends in coronaryevent rates across the who MONICA project populations. Lancet 2000;355:675-687.

11. Rollin A, Maury P, Bongard V, Sacher F, Delay M, Duparc A, Mondoly P, Carrie D, Ferrieres J, Ruidavets JB. Prevalence, prognosis, and identification of the malignant form of early repolarization pattern in a population-based study. Am J Cardiol 2012;110:1302-1308.

12. Ferrieres J, Bongard V, Dallongeville J, Arveiler D, Cottel D, Haas B, Wagner A, Amouyel P, Ruidavets JB. Trends in plasma lipids, lipoproteins and dyslipidaemias in French adults, 19962007. Arch Cardiovasc Dis 2009;102:293-301.

13. Wagner A, Sadoun A, Dallongeville J, Ferrieres J, Amouyel P, Ruidavets JB, Arveiler D. High blood pressure prevalence and control in a middle-aged French population and their associated factors: the MONALISA study. J Hypertens 2011;29:43-50.

14. Panicker GK, Manohar D, Karnad DR, Salvi V, Kothari S, Lokhandwala Y. Early repolarization and short qt interval in healthy subjects. Heart Rhythm 2012;9: 1265-1271.

15. Viskin S, Zeltser D, Ish-Shalom M, Katz A, Glikson M, Justo D, Tekes-Manova D, Belhassen B. Is idiopathic ventricular fibrillation a short QT syndrome? Comparison of QT intervals of patients with idiopathic ventricular fibrillation and healthy controls. Heart Rhythm 2004;1:587-591.

16. Patel RB, Ilkhanoff L, Ng J, Chokshi M, Mouchli A, Chacko SJ, Subacius H, Bhojraj S, Goldberger JJ, Kadish $\mathrm{AH}$. Clinical characteristics and prevalence of early repolarization associated with ventricular arrhythmias following acute ST-elevation myocardial infarction. Am J Cardiol 2012;110:615-620. 
387 Baseline ECG characteristics. Values are given as mean \pm SD or number (percent). VF = ventricular fibrillation.

\begin{tabular}{|c|c|c|c|}
\hline & VF group $(\mathrm{N}=92)$ & Controls $(\mathrm{N}=247)$ & $P$ value \\
\hline Heart rate $(\mathrm{bpm})$ & $69 \pm 16$ & $63 \pm 10$ & .002 \\
\hline PR interval (ms) & $167 \pm 38$ & $168 \pm 23$ & .78 \\
\hline QT interval (ms) & $369 \pm 43$ & $371 \pm 28$ & .70 \\
\hline QTc interval (ms) & $388 \pm 28$ & $377 \pm 20$ & .001 \\
\hline QRS width (ms) & $79 \pm 8$ & $80 \pm 7$ & .65 \\
\hline \multicolumn{4}{|l|}{ Prevalence of $\mathrm{J}$ waves } \\
\hline Inferior leads & $80(87 \%)$ & $184(75 \%)$ & .018 \\
\hline High lateral leads & $29(32 \%)$ & $91(37 \%)$ & .38 \\
\hline Lateral leads & $48(52 \%)$ & $114(46 \%)$ & .33 \\
\hline \multicolumn{4}{|l|}{ J-wave morphology } \\
\hline Only slurred J waves & $27(29 \%)$ & $87(35 \%)$ & .37 \\
\hline Any notched J wave & $65(71 \%)$ & $160(65 \%)$ & \\
\hline \multicolumn{4}{|l|}{ Maximal J-wave amplitude (mV) } \\
\hline Overall & $0.23 \pm 0.11$ & $0.17 \pm 0.07$ & $<.001$ \\
\hline Inferior leads & $0.20 \pm 0.11$ & $0.16 \pm 0.07$ & .004 \\
\hline High lateral leads & $0.16 \pm 0.07$ & $0.14 \pm 0.07$ & .10 \\
\hline Lateral leads & $0.21 \pm 0.10$ & $0.15 \pm 0.07$ & $<.001$ \\
\hline \multirow{2}{*}{\multicolumn{4}{|c|}{ Lead II }} \\
\hline & & & \\
\hline R-wave amplitude (mV) & $1.05 \pm 0.44$ & $0.86 \pm 0.32$ & $<.001$ \\
\hline T-wave amplitude (mV) & $0.22 \pm 0.21$ & $0.30 \pm 0.12$ & $<.001$ \\
\hline $\mathrm{T} / \mathrm{R}$ ratio & $0.23 \pm 0.19$ & $0.37 \pm 0.16$ & $<.001$ \\
\hline ST segment $\leq 0.1 \mathrm{mV}$ & $75(100 \%)$ & $168(99 \%)$ & .57 \\
\hline ST segment $\leq 0.05 \mathrm{mV}$ & $64(85 \%)$ & $134(79 \%)$ & .29 \\
\hline \multicolumn{4}{|l|}{ Lead $V_{5}$} \\
\hline R-wave amplitude (mV) & $1.44 \pm 0.57$ & $1.47 \pm 0.50$ & .68 \\
\hline T-wave amplitude (mV) & $0.29 \pm 0.21$ & $0.47 \pm 0.23$ & $<.001$ \\
\hline $\mathrm{T} / \mathrm{R}$ ratio & $0.20 \pm 0.15$ & $0.33 \pm 0.14$ & $<.001$ \\
\hline ST segment $\leq 0.1 \mathrm{mV}$ & $42(98 \%)$ & $75(77 \%)$ & .003 \\
\hline ST segment $\leq 0.05 \mathrm{mV}$ & $26(61 \%)$ & $37(38 \%)$ & .017 \\
\hline ST segment $\leq 0.1 \mathrm{mV}$ (lead II or $\left.\mathrm{V}_{5}\right)$ & $79(99 \%)$ & $178(88 \%)$ & .004 \\
\hline ST segment $\leq 0.05 \mathrm{mV}$ (lead II or $\mathrm{V}_{5}$ ) & $58(73 \%)$ & $124(61 \%)$ & .10 \\
\hline Lower T/R ratio (lead II or $V_{5}$ ) & $0.18 \pm 0.16$ & $0.30 \pm 0.12$ & $<.001$ \\
\hline
\end{tabular}


392 Performance of various ECG parameters in differentiating malignant and benign inferolateral ER. $\mathrm{Cl}=$

ratio; $\mathrm{OR}=$ diagnostic odds ratio; $\mathrm{SN}$ = sensitivity; $\mathrm{SP}$ = specificity

\begin{tabular}{|c|c|c|c|c|c|c|}
\hline & SN & SP & $+\mathrm{LR}$ & $-\mathrm{LR}$ & OR $(95 \% \mathrm{CI})$ & $P$ value \\
\hline $\begin{array}{l}\text { Presence of inferior ER } \\
\text { Maximal J-wave amplitude }\end{array}$ & $87 \%$ & $26 \%$ & 1.17 & 0.51 & $2.28(1.17-4.46)$ & .018 \\
\hline$>0.2 \mathrm{mV}$ & $46 \%$ & $79 \%$ & 2.17 & 0.69 & $3.15(1.89-5.26)$ & $<.001$ \\
\hline$>0.3 \mathrm{mV}$ & $21 \%$ & $96 \%$ & 5.67 & 0.82 & $6.88(2.99-15.87)$ & $<.001$ \\
\hline \multicolumn{7}{|l|}{ ST segment } \\
\hline$\leq 0.1 \mathrm{mV}\left(\right.$ lead II or $\left.V_{5}\right)$ & $99 \%$ & $12 \%$ & 1.12 & 0.11 & $10.65(1.42-80.12)$ & .004 \\
\hline$\leq 0.05 \mathrm{mV}$ (lead II or $\left.\mathrm{V}_{5}\right)$ & $73 \%$ & $39 \%$ & 1.18 & 0.71 & $1.66(0.94-2.92)$ & 0.10 \\
\hline \multicolumn{7}{|l|}{ QTc interval } \\
\hline$>420 \mathrm{~ms}$ & $20 \%$ & $98 \%$ & 9.67 & 0.82 & $11.77(4.23-32.79)$ & $<.001$ \\
\hline$>400 \mathrm{~ms}$ & $33 \%$ & $88 \%$ & 2.69 & 0.77 & $3.5(1.96-6.25)$ & $<.001$ \\
\hline Presence of low-amplitude T wave & $29 \%$ & $97 \%$ & 9.06 & 0.73 & $12.41(5.38-28.61)$ & $<.001$ \\
\hline \multicolumn{7}{|l|}{ Lower T/R ratio (lead II or $\mathrm{V}_{5}$ ) } \\
\hline$<0.25$ & $77 \%$ & $67 \%$ & 2.35 & 0.34 & 6.93 (3.98-12.07) & $<.001$ \\
\hline$<0.20$ & $61 \%$ & $81 \%$ & 3.13 & 0.49 & $6.45(3.82-10.89)$ & $<.001$ \\
\hline$<0.15$ & $41 \%$ & $89 \%$ & 3.78 & 0.66 & $5.73(3.22-10.20)$ & $<.001$ \\
\hline$<0.10$ & $27 \%$ & $97 \%$ & 8.39 & 0.75 & 11.15 (4.81-25.85) & $<.001$ \\
\hline
\end{tabular}

396

Table 3

Binomial logistic regression model

$95 \%$ Confidence interval for odds ratio

\begin{tabular}{|c|c|c|c|c|c|c|c|c|}
\hline & B & SE & Wald & df & $P$ value & Odds ratio & Lower & Upper \\
\hline QTc interval per $10 \mathrm{~ms}$ & 0.14 & 0.06 & 4.84 & 1 & .028 & 1.15 & 1.02 & 1.30 \\
\hline Maximal J-wave amplitude per $0.1 \mathrm{mV}$ & 0.52 & 0.16 & 10.37 & 1 & .001 & 1.68 & 1.23 & 2.31 \\
\hline Lower $\mathrm{T} / \mathrm{R}$ ratio (lead II or $\mathrm{V}_{5}$ ) per 0.1 unit & -0.48 & 0.14 & 12.30 & 1 & $<.001$ & 0.62 & 0.47 & 0.81 \\
\hline Presence of a dysmorphic T wave & 1.26 & 0.53 & 5.77 & 1 & .016 & 3.53 & 1.26 & 9.88 \\
\hline Presence of $\mathrm{J}$ waves in the inferior leads & 0.95 & 0.40 & 5.64 & 1 & .018 & 2.58 & 1.18 & 5.65 \\
\hline Constant & -7.02 & 2.56 & 7.49 & 1 & .006 & 0.001 & & \\
\hline
\end{tabular}


404

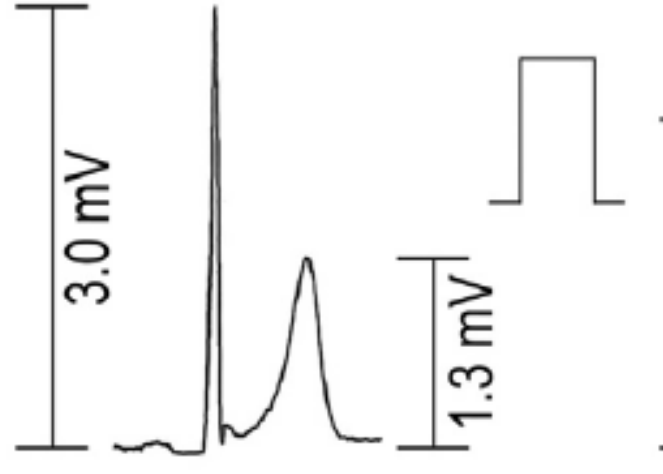

T/R ratio 0.43

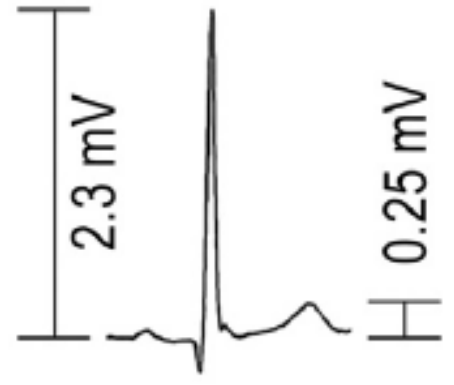

T/R ratio 0.11

406

407

Figure 2

A: Bar graph showing the distribution of QTc interval (in milliseconds) for the ventricular fibrillation 409 (VF)group (green bars) and controls (blue bars). B: Dot plot of the lower T/R ratio (lead II or $V_{5}$ ) for the $410 \quad$ VF group and controls.

411

A

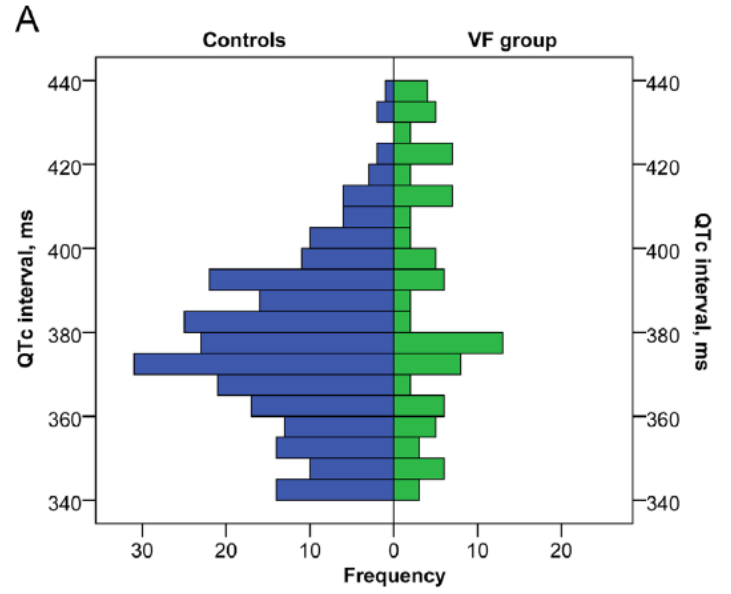

B

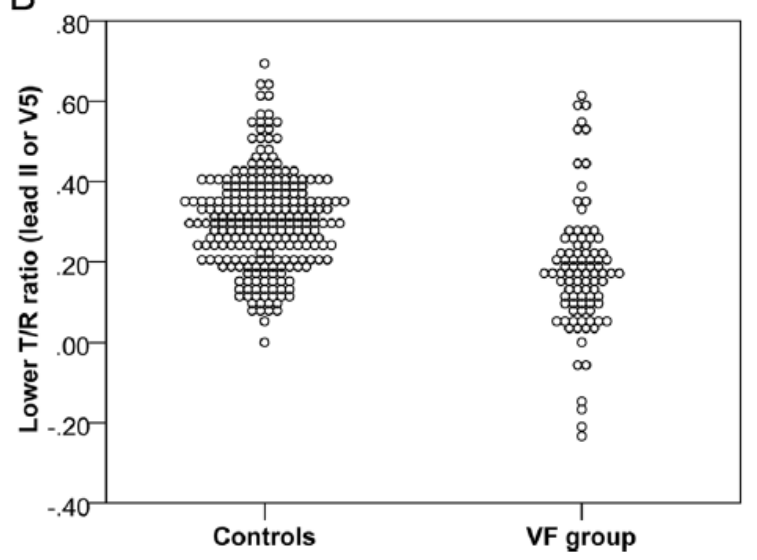


Figure 3

414

ST-segment morphology measured $100 \mathrm{~ms}$ after the J point $(\mathrm{ST} \leq 0.0 \mathrm{mV}, \mathrm{ST} \leq 0.05 \mathrm{mV}, \mathrm{ST} \leq 0.1 \mathrm{mV}$,

415 and ST $>0.1 \mathrm{mV}$ ) and corresponding $\mathrm{T}$-wave amplitudes in leads II and $\mathrm{V}_{5}$ for the ventricular fibrillation

416 (VF) group and controls.

417
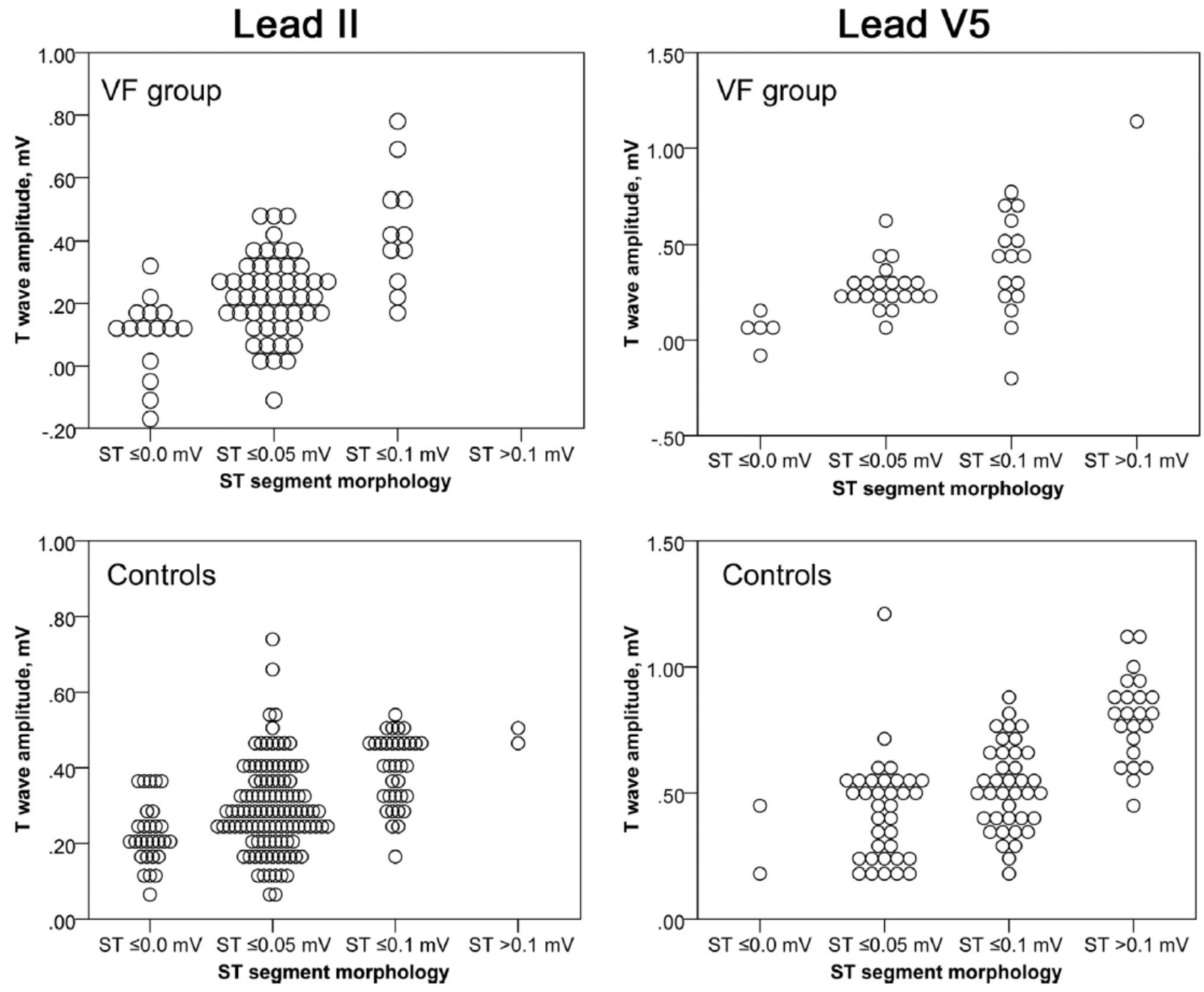
421 Receiver operating characteristic curves for differentiating malignant from benign inferolateral early

422 repolarization based on maximal J-wave amplitude, QTc interval, and lower T/R ratio (lead II or $V_{5}$ ). $\mathrm{AUC}=$ area under receiver operating characteristic curve

424

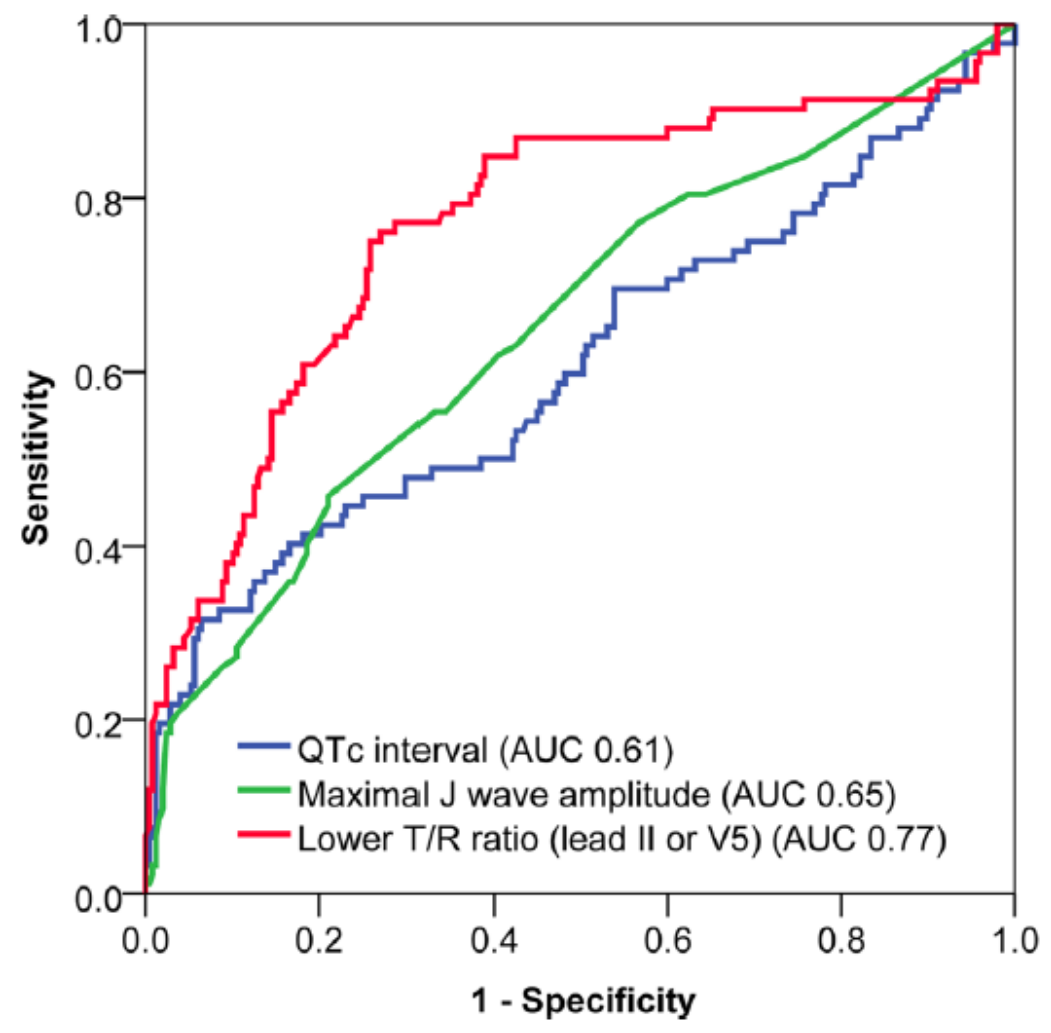


427 ECG examples of inferolateral early repolarization. ECGA: Ventricular fibrillation (VF) group (maximal

428 J-wave amplitude $0.15 \mathrm{mV}$; no dysmorphic T waves; QTc $435 \mathrm{~ms}$; lower T/R ratio 0.11 in lead V 5 ). ECGB:

429 VF group (maximal J-wave amplitude $0.47 \mathrm{mV}$; dysmorphic T wave in lead II; QTc $390 \mathrm{~ms}$; lower T/R

430 ratio 0.08 in lead II). ECG C: Control group (maximal J-wave amplitude $0.3 \mathrm{mV}$; no dysmorphic T waves;

431 QTc 387 ms; lower T/R ratio 0.37 in lead II). ECG D: Control group (maximal J-wave amplitude $0.15 \mathrm{mV}$;

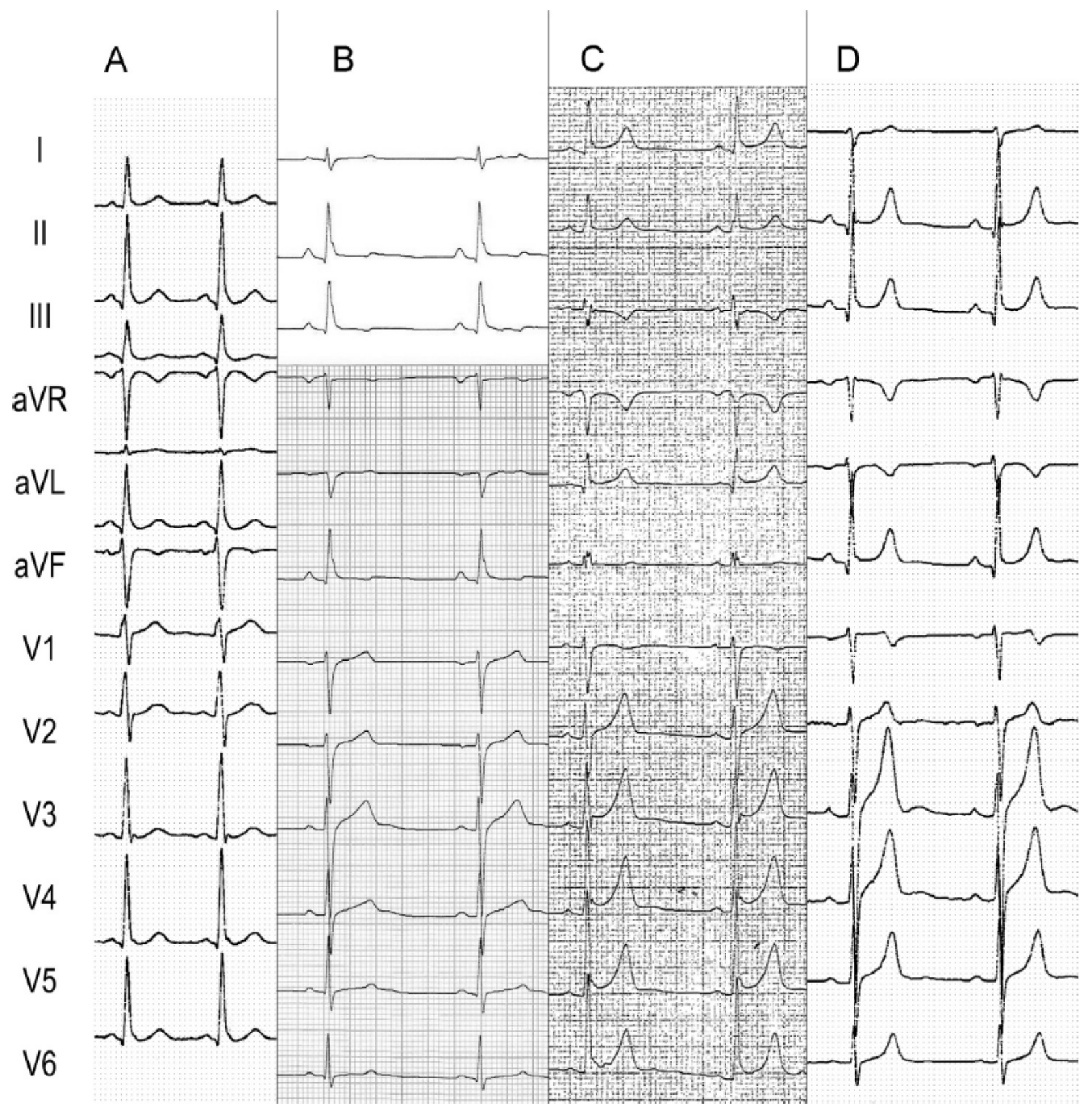

